\title{
Isolated brain metastasis from malignant melanoma of choroid seven years following enucleation
}

Sir,

Choroidal melanoma is the most frequent primary intraocular malignant tumor in adults. Brain metastasis from malignant melanoma of choroid is very rare and is usually noted concomitant with liver metastasis. Authors report a rare case of late isolated brain metastasis from choroidal melanoma seven years after enucleation, which was successfully treated by surgery along with postoperative chemo-radiotherapy.

A 48-year-old male presented to our outpatient services with complaint of headache in left frontal region for preceding six months. He was operated for right eye malignant melanoma of choroid for which enucleation was carried out seven years back. Patient was asymptomatic until the onset of headache six months back and had undergone periodic ophthalmic and systemic evaluation. Patient did not give any history of systemic complaints. 
On examination, he had artificial right eye. Visual acuity of left eye was 6/18. Neurological examination revealed mild upper motor neuron type right-sided hemiparesis.

Routine hematological and biochemical investigations were within normal limits. Ultrasound abdomen, contrast-enhanced computerized tomography (CECT) chest and bone scan did not reveal any evidence of metastasis. Magnetic resonance imaging (MRI) of brain revealed a space-occupying lesion with perilesional edema in left posterior frontal region, which was hyperintense on $\mathrm{T}_{1} \mathrm{~W}$, hypointense on $\mathrm{T}_{2} \mathrm{~W}$ images and showed mild enhancement after injection of intravenous gadolinium [Figure 1a, Figure 1b]. In view of prior history of choroidal melanoma and typical MRI findings, a possibility of brain metastasis from choroidal melanoma was considered and patient was planned for surgery.

Patient underwent left frontal quadrangular flap

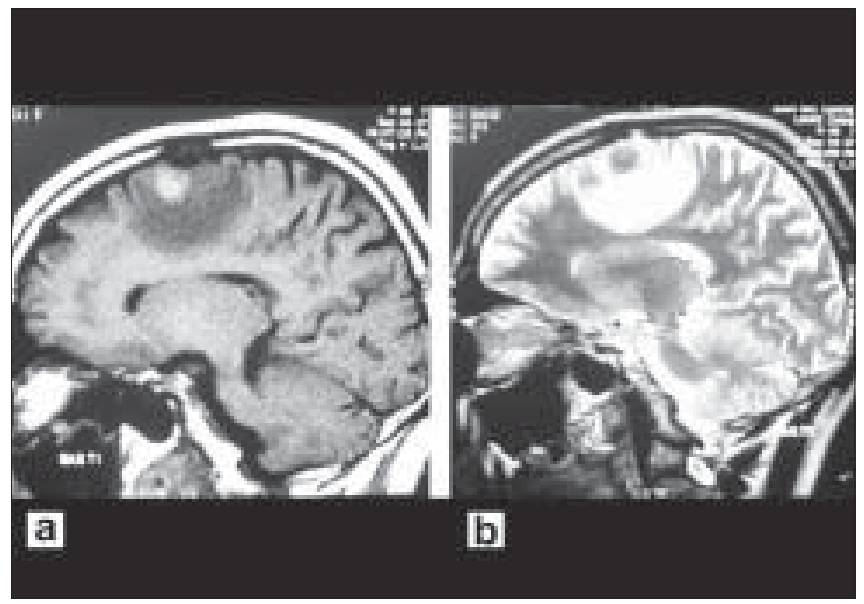

Figure 1a, 1b: Sagittal magnetic resonance imaging (MRI) of brain showing hyperintense lesion on $T_{1} W$ and hypointense lesion on $T_{2} W$ image in posterior frontal region with perilesional edema. The imaging findings are suggestive of melanoma metastasis in view of prior history of choroidal melanoma craniotomy. Intraoperatively, dural metastases were present along with tumor spread in subarachnoid space. There was a blackish grey-white junction soft, suckable tumor suggestive of secondary deposits of malignant melanoma. Gross total excision of the tumor was done along with primary dural closure. He had an uneventful postoperative course and was discharged on fifth postoperative day. Postoperative computerized tomography (CT) brain did not show any evidence of residual tumor. Patient also received postoperative chemotherapy and radiotherapy and was doing well at last follow-up visit nine months after surgery.

Histopathological examination of the excised specimen was suggestive of brain secondary from malignant melanoma of choroid with dense deposit of melanin in the cerebral cortex and Virchow Robbin space along with subpial spread of melanoma cells [Figure 2].

Metastatic uveal melanoma is a highly malignant disease with a limited life expectancy. Liver is the most common site for metastasis of choroidal melanoma followed by lung, bone, skin and subcutaneous tissue.

Metastases from choroidal melanoma usually occur within the first five years of treatment for primary tumor. ${ }^{[1]}$ However, late metastases have been reported up to 40 years after enucleation of primary tumor. ${ }^{[2,3]}$

Metastatic potential of primary tumor depends upon cytological and histological parameters, age of patient at diagnosis, tumor size, tumor location and presence of extrascleral extension. ${ }^{[4]}$ Enucleation has been the standard treatment for ocular melanoma and was used in most reported patients including our case.

The diagnosis of metastatic choroidal melanoma requires close ophthalmological and systemic followup at a regular interval. High index of suspicion along

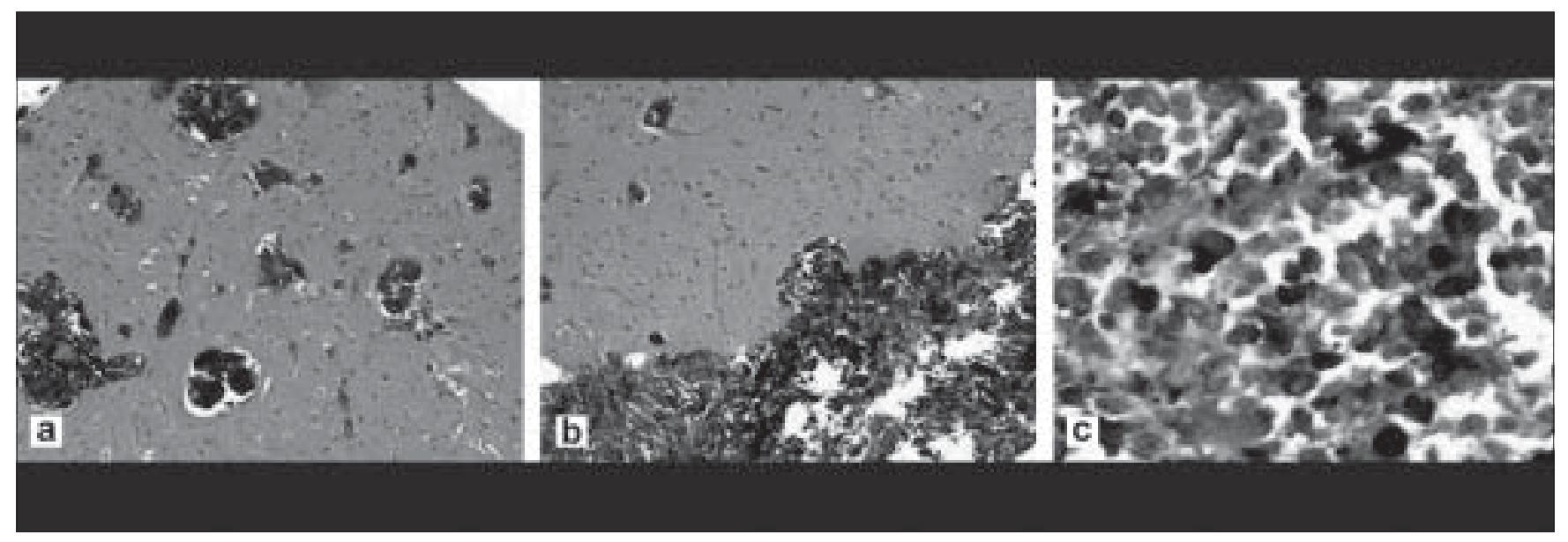

Figure 2: Photomicrograph demonstrates infiltration by a tumor with dense deposit of melanin, in cerebral cortex and Virchow Robbin's space along with subpial spread of melanoma cells (H\&E, $\times 40,100,200)$ 
with typical MRI findings help to confirm the diagnosis. Melanin produces stable free radicals, causing paramagnetic effect that shortens $\mathrm{T}_{1}$ and $\mathrm{T}_{2}$ relaxation values on MRI. This results in hyperintensity of the lesion on $\mathrm{T}_{1} \mathrm{~W}$ and hypointensity on $\mathrm{T}_{2} \mathrm{~W}$ images. ${ }^{[5]}$

Treatment of isolated brain metastasis from malignant melanoma includes total surgical excision of the lesion followed by postoperative adjuvant radiotherapy and chemotherapy as in our case. In spite of all the possible management, long-term prognosis for metastatic choroidal melanoma remains poor.

\section{Sachin A. Borkar, G. D. Satyarthee, Prasenjit Das ${ }^{1}$, Vaishali Suri ${ }^{1}$ \\ Departments of Neurosurgery and ${ }^{1}$ Neuropathology, All India Institute of Medical Sciences, New Delhi, India. \\ E-mail: duttaguru2002@yahoo.com}

\section{References}

DOI: $10.4103 / 0028-3886.48798$

1. Lorigan JG, Wallace S, Mavligit GM. The prevalence and location of metastases from ocular melanoma: Imaging study in 110 patients. AJR Am J Roentgenol 1991;157:1279-81.

2. Abramson DH, Servodidio CA. Metastatic choroidal melanoma to the contralateral orbit 40 years after enucleation. Arch Ophthalmol $1997 ; 115: 134$.

3. Achtaropoulos AK, Mitsos AP, Detorakis ET, Georgakoulias NV, Drakonaki EE, Kozobolis VP. Late isolated brain metastasis following enucleation for choroidal melanoma. Ophthalmic Surg Lasers Imaging 2005;36:151-4

4. Zimmerman LE, McLean IW. The pathogenesis of metastases from uveal melanomas. Ophthalmic Forum 1983;1:28-9.

5. Gomori JM, Grossman RI, Shields JA, Ausberger JJ, Joseph PM, DeSemione D. Choroidal melanomas: Correlation of NMR spectroscopy and MR imaging. Radiology 1986;158:443-5.

Accepted on 06-02-2009 